\title{
Spatial regularity of the young stellar population in the ring of NGC 6217
}

\author{
Alexander S. Gusev and Elena V. Shimanovskaya
}

\begin{abstract}
Sternberg Astronomical Institute, Lomonosov Moscow State University, Universitetsky pr. 13, 119234 Moscow, Russia e-mail: gusev@sai.msu.ru
\end{abstract}

Received 29 May 2020 / Accepted 15 July 2020

\begin{abstract}
The relative contribution of various physical processes to the spatial and temporal distribution of molecular clouds and star-forming regions in the disks of galaxies has not yet been the subject of extensive study. Investigating the spatial regularity in the distribution of the young stellar population in spiral and ring structures is a good test for studying this contribution. In this paper, we look at the photometric properties of the ring and spiral arms in the barred spiral galaxy NGC 6217 based on an analysis using GALEX ultraviolet, optical $U B V R I$, and $\mathrm{H} \alpha$ surface photometry data. The ring in the galaxy is located near the corotation area. We found evidence of spatial regularity in the distribution of the young stellar population along the galaxy ring. The characteristic scale of spacing is about $700 \mathrm{pc}$. At the same time, we did not find a similar regularity in the distribution of the young stellar population along the spiral arms of NGC 6217. The spatial regularity in the concentration of young stellar groupings along spiral arms is a quite rare phenomenon and it has never previously been seen in galactic rings.
\end{abstract}

Key words. galaxies: individual: NGC 6217 - galaxies: star formation - galaxies: ISM

\section{Introduction}

Various physical processes, such as gravitational collapse and turbulence compression, are believed to play a main role in the creation of molecular clouds and their further evolution into star formation regions. The sizes of these structures and their spatial distribution in galactic disks can be explained in terms of gravitational or magnetogravitational instability (see, e.g., Elmegreen \& Elmegreen 1983; Elmegreen 1994b). However, the influence of the magnetic field and shock waves on the spatial distribution of gas clouds and young stellar groups is still not fully understood. Galactic spiral arms and rings are good laboratories for studying the relative effect of different physical processes on the spatial distribution of star-forming regions.

The regular spatial distribution of young star complexes is a rather unique phenomenon which was visually detected by Elmegreen \& Elmegreen (1983) in spiral arms of only 22 grand design galaxies. We note that among these galaxies, present in only seven stellar systems, the regular chains of star complexes are observed in both spiral arms. The data of Elmegreen \& Elmegreen (1983) remains the only published result of a systematic search for regular chains of stellar complexes in spiral arms.

In later years, Efremov $(2009,2010,2011)$ found the regularities in the distribution of H II superclouds in spiral arms of our Galaxy and in the distribution of star complexes in M31. Gusev \& Efremov (2013) confirmed the result of Elmegreen \& Elmegreen (1983), finding that only one of the spiral arms in NGC 628 has a regular chain of star complexes with a separation of $\sim 1.7 \mathrm{kpc}$. However, they also found the existence of the same characteristic separation $(\approx 400 \mathrm{pc})$ between adjacent fainter star-formation regions in both spiral arms of the galaxy. Finally, Elmegreen et al. (2018) found regularly spaced infrared peaks in the dusty spiral arms of M 100 with a typical spacing between the clumps of $\sim 410 \mathrm{pc}$

Along with spiral arms, galactic rings are the product of evolutionary processes taking place in galactic disks. These appear to be related to specific orbital resonances with the pattern speed of a bar (Buta 2017). Unlike arms, the physical and dynamic parameters of medium (gas density, temperature, pressure, rotation velocity) along rings are likely to be constant. On the other hand, strong shock waves are not expected to be present in rings. These features make them interesting objects for the study of the spatial distribution of star-forming regions.

Based on general ideas about physics and evolution of rings, investigating spatial regularity in the distribution of star clusters, as well as in arms, is not an impossible undertaking. Elmegreen (1994a) theoretically described the possibility of the existence of regularly spaced starburst spots in galactic rings. However, this had not been seen in observations before. Only Artamonov et al. (1999) noted quasi-periodic characteristic distances of $1.3 \pm 0.2 \mathrm{kpc}$ between adjacent star complexes in the ringed barred galaxy NGC 6217. The purpose of our study is to verify the possible regularity in the distribution of star formation regions along the ring of NGC 6217.

NGC 6217 is a galaxy with a prominent bar, two strong spiral arms, and two rings. One peculiarity of the galaxy is that its spiral arms begin from the nuclear region and, upon crossing the ends of the bar, they form a (pseudo-)ring with a major projected radius of 40.2 arcsec (Comerón et al. 2014) (Fig. 1). The faint outer ring has radius that is $\approx 2$ times larger then the inner one (81.6 arcsec according to Comerón et al. 2014). 


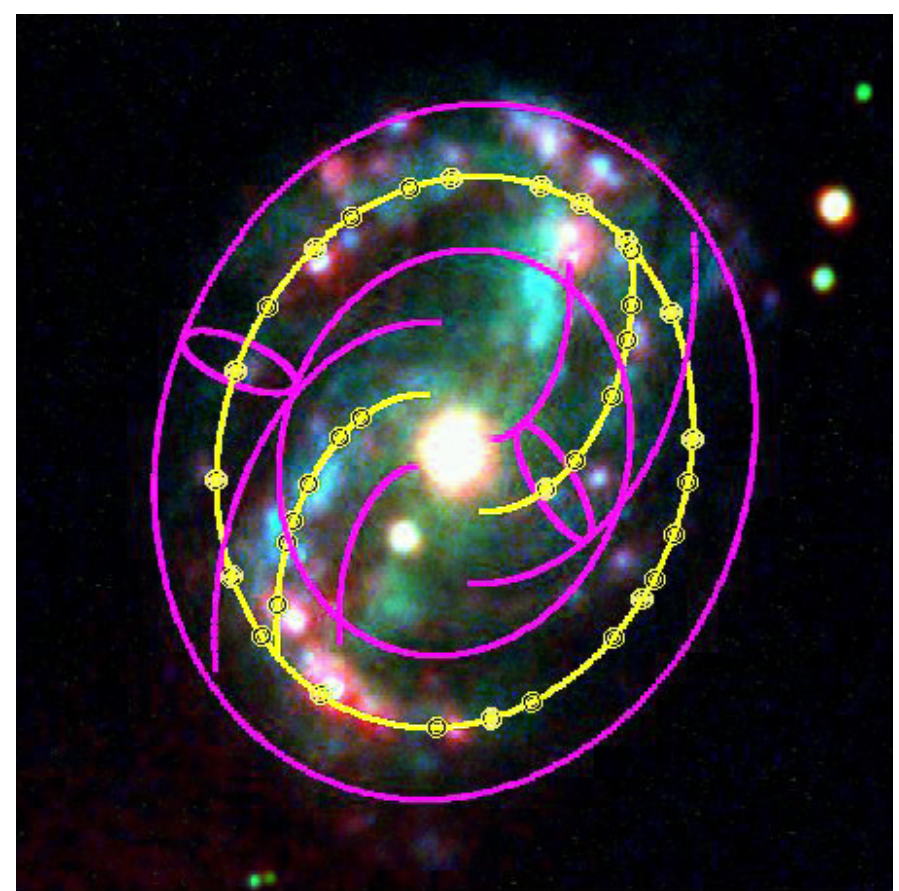

Fig. 1. Composite image of NGC 6217 in the $U$ (blue), $B$ (green) passbands, and $\mathrm{H} \alpha$ line (red) with the logarithmic spiral segments and the ring (yellow lines) overlaid. The yellow-black-yellow circles on the curves of the arm and ring correspond to the projected positions of the local maxima of brightness. The areas of flux measurements in apertures are bounded by magenta ellipses (segments). Examples of elliptical apertures are shown in magenta. The size of the image is $120 \times 120 \mathrm{arcsec}^{2}$. North is upward and east is to the left.

However, it is not considered in this paper and so, it is not shown in Fig. 1.

The photometry and kinematics of the galaxy have been studied in a wide range of wavelengths from UV to radio (see van Driel \& Buta 1991; Elmegreen et al. 1996; Artamonov et al. 1999; James et al. 2004; Hernandez et al. 2005; Garrido et al. 2005; Font et al. 2019, and references therein). We note that the NGC 6217 inner ring that is the subject of current studies is located near the corotation region (van Driel \& Buta 1991; Elmegreen et al. 1996; Font et al. 2014).

The fundamental parameters of NGC 6217 are presented in Table 1, where the morphological type is taken from Buta et al. (2014), Galactic absorption, $A(B)_{\mathrm{Gal}}$, taken from the NED ${ }^{1}$ database, and the remaining parameters are taken from the LEDA $^{2}$ data base. The adopted value of the Hubble constant is equal to $H_{0}=75 \mathrm{~km} \mathrm{~s}^{-1} \mathrm{Mpc}^{-1}$. With the assumed distance to NGC 6217, we estimate a linear scale of $100 \mathrm{pc} \mathrm{arcsec}^{-1}$. We note that the kinematic positional angle of the galaxy $\left(287^{\circ}\right)$ is significantly different from the photometric one (Font et al. 2019). However, given the low inclination of NGC 6217, this does not substantially affect our results.

\section{Observational data}

The results of the $U B V R I$ photometry for the galaxy were published in Gusev et al. (2015). We also use the $\mathrm{H} \alpha$ image, obtained in the GHASP survey (Epinat et al. 2008) and downloaded from the NED data base, in our study. The absolute calibration of this

\footnotetext{
1 http://ned.ipac.caltech.edu/

2 http://leda.univ-lyon $1 . \mathrm{fr} /$
}

Table 1. Basic parameters of NGC 6217

\begin{tabular}{lc}
\hline \hline Parameter & Value \\
\hline Type & $\left(\mathrm{R}^{\prime} \mathrm{L}\right) \mathrm{SB}(\mathrm{rs}) \mathrm{b}$ \\
Total apparent $B$ magnitude $\left(B_{t}\right)$ & $11.8 \mathrm{mag}$ \\
Absolute $B$ magnitude $\left(M_{B}\right)^{(a)}$ & $-20.45 \mathrm{mag}$ \\
Inclination $(i)$ & $33^{\circ}$ \\
Position angle (PA) & $162^{\circ}$ \\
Apparent corrected radius $\left(D_{25}\right)^{(b)}$ & $2.30 \mathrm{arcmin}$ \\
Apparent corrected radius $\left(D_{25}\right)^{(b)}$ & $13.8 \mathrm{kpc}$ \\
Distance $(d)$ & $20.6 \mathrm{Mpc}$ \\
Galactic absorption $\left(A(B)_{\mathrm{Gal}}\right)$ & $0.158 \mathrm{mag}$ \\
\hline
\end{tabular}

Notes. ${ }^{(a)}$ Absolute magnitude of a galaxy corrected for Galactic extinction and inclination effect. ${ }^{(b)}$ Isophotal diameter $\left(25 \mathrm{mag} \mathrm{arcsec}^{-2}\right.$ in the $B$-band) corrected for Galactic extinction and absorption due to the inclination of NGC 6217.

image was carried out using the parameters of descriptors from the original FITS file (image) and their explanations in Epinat et al. (2008). We already used $\mathrm{H} \alpha$ data for NGC 6217 in Gusev et al. (2018).

Ultraviolet GALEX NUV-reduced FITS-images of NGC 6217 were downloaded from the B. A. Miculski Archive for space telescopes ${ }^{3}$ The observations were carried out on November 11, 2011, with a total exposure of $96 \mathrm{~s}$. The description of the GALEX mission, parameters of NUV passband, files description, and data reduction are presented in Morrissey et al. (2005).

Images of the galaxy in all bands were transformed to the face-on position using the values of $i$ and the position angle (PA) from Table 1. Those face-on images were used for the further analysis. Photometric magnitudes are corrected for Galactic extinction and for projection effects: $m_{\text {cor }}=m_{\text {obs }} 10^{0.4 A_{\text {Gal }}} \cos (i)$. Our image resolution is equal to $1.7 \operatorname{arcsec}$ for $U, 1.4 \operatorname{arcsec}$ for $B$ and $V, 3.3$ arcsec for $\mathrm{H} \alpha$, and 5.3 arcsec for NUV.

\section{Results}

\subsection{Along-ring and-arm photometry}

For the next steps in our analysis, we used a technique developed by us in Gusev \& Efremov (2013). In the first step, we define the geometric parameters of the spiral arms and the ring by fitting the curves of both main arms and the ring, which are clearly outlined in the optical bands (see Fig. 1). The arms and the ring are defined by a visual selection of pixels in the part of these images that belongs to the center lines of the spiral arms or the ring. Pixels in this region are then fitted with a logarithmic spiral for the arms or a circle for the ring using linear least-squares. Here we assume that the pitch angle $\mu$ is constant along the arm and equal for both spirals.

Using the classic equation for the logarithmic spiral $r=$ $r_{0} \exp \left(k\left(\theta-\theta_{0}\right)\right)$, where $k=\tan \mu$, we obtained the following parameters for the spiral arms, $r_{0}=9.65 \operatorname{arcsec}(960 \mathrm{pc})$ and $k=$ $0.75 \pm 0.07$, which corresponds to a pitch angle $\mu=36.8 \pm 2.5^{\circ}$. The zero point angle $\theta_{0}=30^{\circ}$ for the eastern arm and $210^{\circ}$ for the western arm (where $\theta$ is counted from north towards east, the same as the PA; see Fig. 1). As a result of the fitting, we also obtained a radius of the ring $r_{\text {ring }}=38 \pm 4 \operatorname{arcsec}(3.8 \pm 0.4 \mathrm{kpc})$. The ring parameters used by us ( $r$ and PA) are close to those

\footnotetext{
http://galex.stsci.edu/GR6/; files AIS_21_sg76-nd-*.fits.
} 


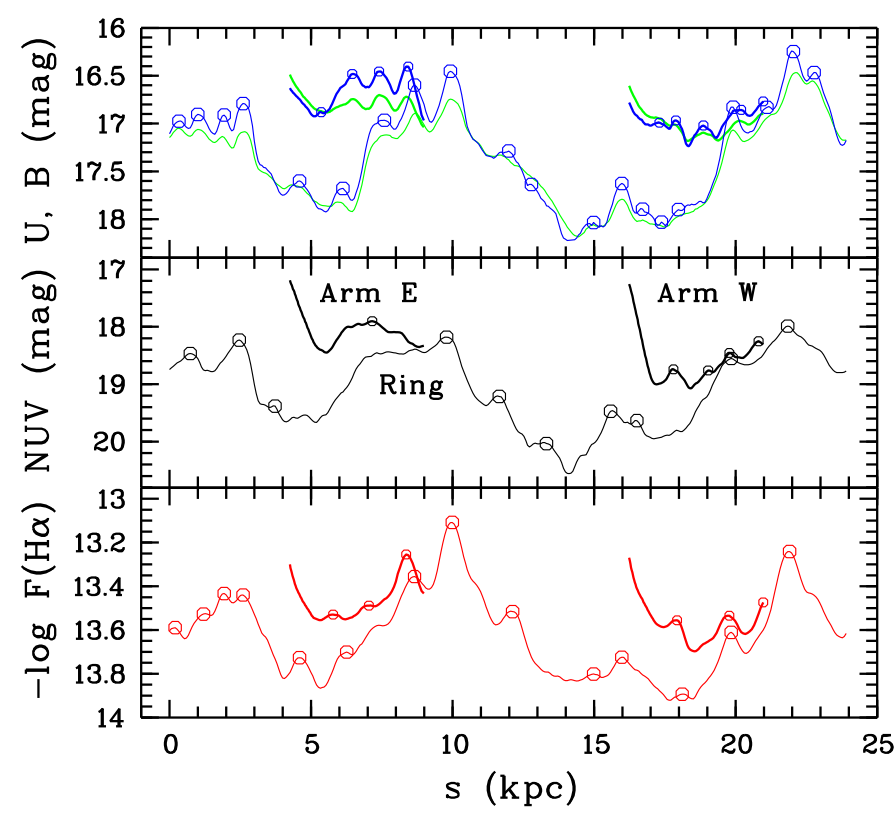

Fig. 2. Photometric profiles in the $U$ (blue), $B$ (green), NUV (black) bands, and $\mathrm{H} \alpha$ line (red) along the ring (thin curves) and the spiral arms (thick curves). The ordinate units are magnitudes and logarithm of $\mathrm{H} \alpha$ flux in units of erg s $\mathrm{s}^{-1} \mathrm{~cm}^{-2}$ within the aperture. Positions of local maxima of brightness on the profiles in the $U$, NUV bands, and $\mathrm{H} \alpha$ line are indicated by circles for the ring (large symbols) and for the arms (small symbols).

obtained by Comerón et al. (2014). The resulting spiral arms and the ring are shown in Fig. 1 in projection to the apparent plane of the galaxy.

We note the extremely high pitch angle, which is $\approx 2$ times larger than the average value for galaxies of the same morphological type (Díaz-Garcíaa et al. 2019). Apparently, this is a consequence of the high central-mass concentration in NGC 6217. It is known that the galaxy has a very bright nucleus in a wide range of wavelengths (see Williams et al. 2019, and references therein), a high H I central surface density (van Driel \& Buta 1991), and it exhibits signs of nuclear activity (Véron-Cetty \& Véron 2010).

To study variations in brightness along the ring and spiral arms, we obtained photometric profiles along these structures. We used the elliptical aperture $\left(20 \times 6 \operatorname{arcsec}^{2}\right)$, with a minor axis along the spiral arm and ring (see Fig. 1) with a step of $1^{\circ}$ by PA This step corresponds to a linear distance of $\approx 67 \mathrm{pc}$ for the ring and varies from $\approx 20$ to $\approx 80 \mathrm{pc}$ for the spiral arms. The strip of 20 arcsec wide captures all star-forming regions in the ring (spiral arms), but does not include areas outside them. The exception is the large star complex in the northwestern part of the bar (Fig. 1), however, in the analysis it is superimposed on the young stellar region in the ring having the same PA. The obtained photometric profiles in NUV, $B, V$, and $\mathrm{H} \alpha$ along the ring and arms are presented in Fig. 2.

\subsection{Spatial regularity of young stellar population along the ring and arms}

Longitudinal displacement along the ring, denoted as $s$, is equal to $r_{\text {ring }}\left(\theta-\theta_{0}\right)$, where $\theta$ is in radians. For the logarithmic spirals, $s=(\sin \mu)^{-1} r_{0}\left(\exp \left(k\left(\theta-\theta_{0}\right)\right)-1\right)$. We adopted a zero-point for the displacement along the ring, $s=0$, corresponding to $\mathrm{PA}=$ 0 . It increases from north toward east, the same as the PA. The

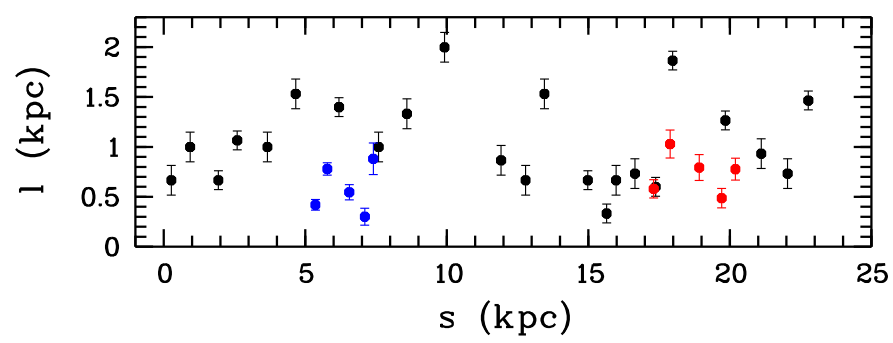

Fig. 3. Separations $l$ between adjacent local maxima of brightness along the ring (black circles), Arm E (blue), and Arm W (red). The error bars are shown. Positioning errors, $\Delta s$, are smaller than the circles size. See the text for details.

displacement values along the spiral arms were combined with the displacements along the ring: at the points of intersection between the spiral arms and the ring at the outer ends of the arms, their displacement was taken equal to the displacement along the ring, $s_{\text {arm }}=s_{\text {ring }}$.

Using mostly profiles in the $U$ band and $\mathrm{H} \alpha$ line, and involving profiles in the NUV band, we found the local brightness maxima on the profiles. We prefer to use the $U$ band image, since it has sufficient resolution and is sensitive to the presence of a young stellar population. In addition, the $\mathrm{H} \alpha$-image was used to identify $\mathrm{H}$ II regions those are weakly visible in the $U$ passband. The low-resolution NUV image, as well as the image in the $B$ band, were used for control.

The local maxima of brightness were determined as the lower extrema of the functions $m_{U}(s), m_{\mathrm{NUV}}(s),-\log F(\mathrm{H} \alpha)(s)$ for the ring and both spiral arms. To locate them, we looked for points with the first derivative of the function, $\mathrm{d} m / \mathrm{d} s=0$ or $\mathrm{d}(\log F(\mathrm{H} \alpha)) / \mathrm{d} s=0$, and the second derivative, $\mathrm{d}^{2} m_{U} / \mathrm{d} s^{2}>0$ or $\mathrm{d}^{2}(-\log F(\mathrm{H} \alpha)) / \mathrm{d} s^{2}=0$, on the profiles. We selected peaks whose widths exceed three measurement points (corresponding to the angular resolution of the images in optical bands) and whose amplitude exceeds 0.043 mag for the $U, 0.017$ dex for $\mathrm{H} \alpha$ and 0.24 mag for the NUV (which corresponds to the threshold $3 \sigma$ above the average background level in the corresponding images within the used aperture, $20 \times 6 \operatorname{arcsec}^{2}$ ).

As a result, we obtained 23 local maxima of brightness in the ring, with 6 maxima in the eastern arm (Arm E), and 6 maxima in the western arm (Arm W). The positions of these maxima are shown in Fig. 2. Some local maxima of brightness do not appear in $U$ or in $\mathrm{H} \alpha$ (see Fig. 2), which is a reflection of the photometric evolution of young star clusters with an age from $\sim 3$ to $\sim 300$ Myr (Whitmore et al. 2011). The typical errors of maximum positions are $\Delta s= \pm 1$ measurement points, which corresponds to an error of $\pm 70 \mathrm{pc}$ for the ring; the maximum $\Delta s$ does not exceed $140 \mathrm{pc}$ (see Fig. 3).

In the next step, we measure separations, $l$, between adjacent local maxima of brightness along the ring and arms (Fig. 3). The separation between the $n$th and $(n+1)$ st maxima is defined as $l_{n}=$ $s_{n+1}-s_{n}$. For the ring, we looped the profile so that $l_{23}=s_{1}-s_{23}$. Figure 3 shows a rather regular separation between adjacent local maxima of brightness along the ring.

We built a histogram of the distribution of separations $l$ in the ring and in the spiral arms (Fig. 4). We note that the arm distributions do not show noticeable regularity. For the ring, the histogram shows a well-pronounced main peak in the distribution at $l \approx 700 \mathrm{pc}$ and several secondary peaks.

We estimated the mean and median separations between local maxima of brightness in the ring of the galaxy for four separate subsets of objects, numbered in Fig. 4. Our results are 


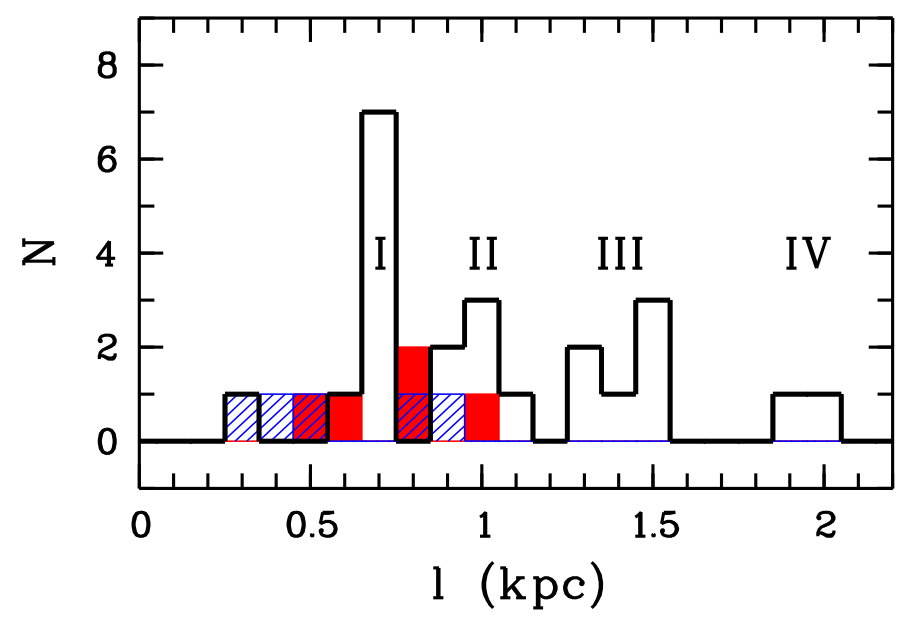

Fig. 4. The number distribution histograms of local maxima of brightness by separation between adjacent objects along the ring (thick black line), Arm E (shaded in blue), and Arm W (filled red). Roman numerals denote subsets of the objects with close characteristic separations.

Table 2. Characteristic separations $l$ of local maxima of brightness in the ring.

\begin{tabular}{lccc}
\hline \hline Subset & $N$ & Mean $(\mathrm{kpc})$ & Median $(\mathrm{kpc})$ \\
\hline I & 8 & $0.67 \pm 0.04$ & 0.67 \\
II & 6 & $0.98 \pm 0.07$ & 1.00 \\
III & 6 & $1.42 \pm 0.11$ & 1.40 \\
IV & 2 & $1.93 \pm 0.09$ & 1.86 \\
\hline
\end{tabular}

presented in Table 2, where the confidence intervals correspond to a $95 \%$ confidence probability $(0.05$ significance level in the framework of Student's $t$-test). The errors in the table do not include the intrinsic measurement errors in pairs, that is, equal to $\approx 0.1 \mathrm{kpc}$, which does not depend on the affiliation of pairs to a particular subset.

We also note that there is no correlation between the affiliation of pairs of local maxima of brightness to different subsets and the accuracy of measurement of their positions and peak amplitudes.

Thus, the distribution of separations can be multimodal. Given the sample size is too small to make a robust multimodality test, we calculated distances $D$ between the peaks relative to their widths according to the definition by Ashman et al. (1994): $D=\left|\mu_{a}-\mu_{b}\right| / \sqrt{\left(\sigma_{a}^{2}+\sigma_{b}^{2}\right) / 2}$, where $\mu_{a}, \mu_{b}$ are the means of subsets $a$ and $b$ (I and II or II and III), $\sigma_{a}, \sigma_{b}$ are the standard deviations. For both pairs of subsets $D>4$, as well as for a mixture of two Gaussian distributions, $D>2$ is required for a clean separation between the modes. Additionally, we calculated distances between peaks and check if they exceed $3 \sigma_{\text {tot }}$ (where $\sigma_{\text {tot }}$ comprises both statistical errors and measurement errors for a pair of subsets): $\Delta \mu_{\mathrm{I}-\mathrm{II}}=0.31 \mathrm{kpc}$, whereas $3 \sigma_{\mathrm{I}-\mathrm{II}}=0.29 \mathrm{kpc}$; and $\Delta \mu_{\mathrm{II}-\mathrm{III}}=0.44 \mathrm{kpc}$, whereas $3 \sigma_{\mathrm{II}-\mathrm{III}}=0.42 \mathrm{kpc}$.

The most important result obtained from the analysis of the distribution over $l$ is the multiplicity of secondary peaks relative to the main one. The characteristic (mean) separations in subset II are about one and a half times greater $(1.46 \pm 0.25$, considering the internal positioning errors of pairs), while in subset III, they are about two times greater $(2.12 \pm 0.36)$, and in subset IV, they are about three times greater $(2.88 \pm 0.49)$ than the separation in subset I. We do not discuss subset IV as it contains only two measurements, which is insufficient for a statistic analysis.
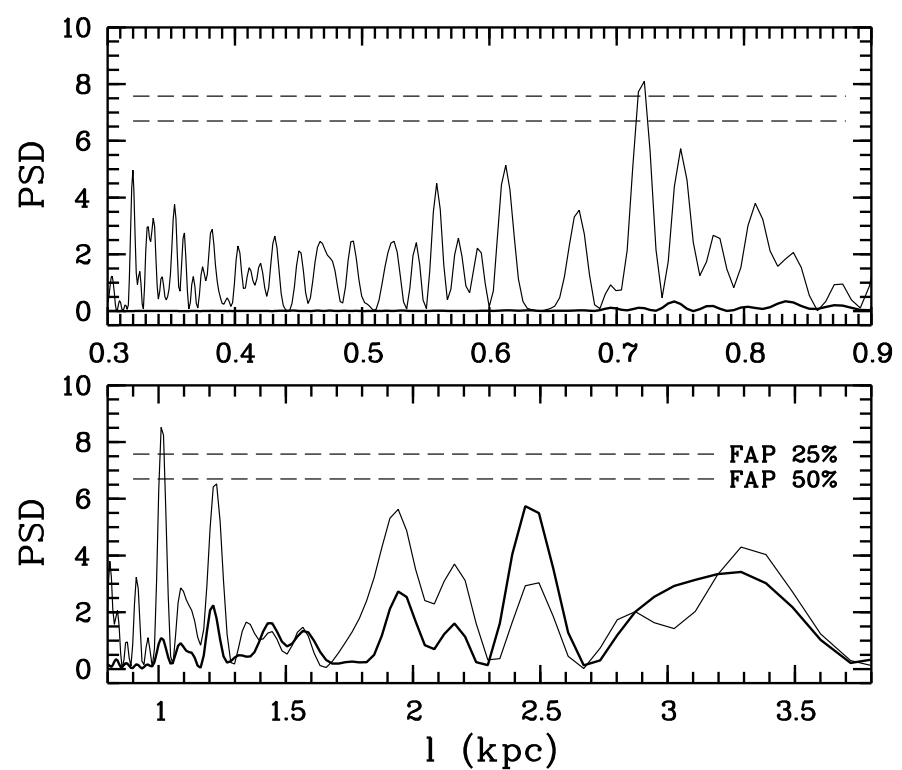

Fig. 5. Normalized power spectral density of the $U$ profile data from Fig. 2 (thick curves) and the function $p(s)$ (thin curves) for the ring. Dashed lines are the FAP levels of 25 and 50\%. See the text for more explanation.

Additionally, to estimate the power spectrum of our data, we computed the Lomb-Scargle periodograms for the $U$-band profiles along the ring shown in Fig. 2, and for the function $p(s)$. The function $p(s)$ is a collection of Gaussians, centered at points of local maxima of brightness on the profiles, with $\sigma$ equal to the peak positioning error. The Gaussian amplitudes are assumed to be equal to the measured peak amplitudes, normalized to the maximum amplitude. If significant peaks were recorded in several bands ( $U, \mathrm{NUV}, \mathrm{H} \alpha$ ), the largest normalized amplitude was adopted. Outside of the Gaussians, the function $p(s)=0$ is valid for all other points.

The periodograms, obtained using the technique developed by Scargle (1982), Horne \& Baliunas (1986), and Press \& Rybicki (1989), also thoroughly explained by VanderPlas (2018), are presented in Fig. 5. The Fourier analysis data, obtained for $U$ profiles and for functions $p(s)$, complement each other to support the presence of the spatial regularity of local maxima of brightness. The former shows characteristic low frequencies $(l>2 \mathrm{kpc}$, bottom panel in Fig. 5), the latter represents high frequencies $(l<$ $2 \mathrm{kpc}$, upper panel).

The periodograms have noticeable peaks with a false-alarm probability <25\% (FAP; see Horne \& Baliunas 1986, for details) of $l \approx 0.72$ and $\approx 1.0 \mathrm{kpc}$, which corresponds to the characteristic separations in subsets I and II, respectively. Hence, the results of the Fourier analysis support the estimation of characteristic separations of local maxima of brightness in the ring based on their distributions in Figs. 3 and 4 and also presented in Table 2.

\section{Discussion}

Earlier in this paper, we use NGC 628 as an example to discuss the effect of a shock wave on the formation of regularity in the distribution of young stellar groupings, along with a possible anti-correlation between shock wave signatures and the presence of chains of star complexes (Gusev \& Efremov 2013). This galaxy has two primary spiral arms, one of them showing an absence of shock wave and hosting the regular chain of star complexes, while the other does not. Nevertheless, both spiral 
arms in NGC 628 have the same regular characteristic separation between fainter star-formation regions that are adjacent to one another.

NGC 6217 is an example of a galaxy in which a similar regularity in the distribution of young stellar groups occurs in the ring located near the corotation radius. No shock waves are expected to be observed in this area. The detection of regularity here is a rather unusual result. In the corotation region, $\mathrm{HI} / \mathrm{H}_{2}$ clouds, the progenitors of $\mathrm{H}$ II regions and young stellar groups are not expected to experience any significant mutual influence and they do not show a tendency towards concentration. A possible reason for the structural features observed in the ring of NGC 6217 may be that it may be, in fact, a pseudo-ring. A prominent bar can act as the driver of the Jeans instability wave. We note that the largest distances between adjacent maxima of brightness are observed directly in front of the bar (relative to the direction of rotation of the galaxy; see Fig. 1).

We obtained the characteristic distance, $\Lambda \sim 700 \mathrm{pc}$, which seems to correspond to a double wavelength of spacing regularity. Elmegreen et al. (2018) in M 100 and Gusev \& Efremov (2013) in M74 (NGC 628) obtained the characteristic spacings of $\sim 400 \mathrm{pc}$, half as large. Our subset II corresponds to $3 / 2 \Lambda$, which may indicate the existence of a wavelength of $1 / 2 \Lambda$. The power spectral density of the $U$ profile has the largest peak at $l=2.45 \mathrm{kpc}$ (Fig. 5) that is close to $7 / 2 \Lambda$. Our linear resolution is insufficient to look for spatial regularities on the scale of $1 / 2 \Lambda$.

The characteristic spacing wavelength for hydrogen clouds is $\lambda_{0}=2 c^{2}(G \sigma)^{-1}$, where $c$ is the sound speed and $\sigma$ is the mass column density of the gas (Elmegreen \& Elmegreen 1983). Spatial regularity in the ring of NGC 6217 indicates the constancy of parameters of the gas medium along the ring. The mass column density of H I near the ring is $\sigma \approx 5 M_{\odot} \mathrm{pc}^{-2}$ (van Driel \& Buta 1991). The sound speed $c$ is a few $\mathrm{km} \mathrm{s}^{-1}$ for both $\lambda_{0}=\Lambda$ and $\lambda_{0}=1 / 2 \Lambda$, which is a typical speed in a diffuse H I medium.

\section{Conclusions}

For the first time, a spatial regularity was found in the distribution of the young stellar population in the galaxy ring located near the corotation region. This refines our understanding of the physical processes that contribute to the formation of a regular wave of star formation in rings and arms. The presence or absence of shock waves does not appear to affect the genesis of regularity in regions of star formation.

The obtained characteristic separation, $\Lambda$, between adjacent young stellar groupings in the ring of NGC 6217 is equal to $\approx 670 \mathrm{pc}$, that is twice as much as the characteristic separation obtained for young stellar groups in the spiral arms of M 100 and $M 74$. However, we suspect a characteristic separation with a scale of $1 / 2 \Lambda$ along the ring of NGC 6217 , the same as was found earlier in spiral arms of M 100 and M 74. This may indicate a unified mechanism for the formation of such regularities in spiral arms and in the rings of galaxies. Achieving greater clarity around the characteristic separations in the rings requires the search and analysis of regularities in nearer ringed galaxies with improved spatial resolution.

Acknowledgements. We are grateful to the referee for their constructive comments. We would like to honor Prof. Yu. N. Efremov, who was the initiator of this research program, and who left us in August 2019. The authors acknowledge the use of the HyperLeda data base (http://leda.univ-lyon1.fr), the NASA/IPAC Extragalactic Database (http://ned.ipac.caltech.edu), Barbara A. Miculski Archive for space telescopes (https://archive.stsci. edu), and the IDL Astronomy User's Library (https://idlastro.gsfc. nasa.gov). This study was supported by the Russian Foundation for Basic Research (project no. 20-02-00080). A.G. acknowledges the support from the Lomonosov Moscow State University Development Program (Leading Scientific School "Physics of stars, relativistic objects and galaxies").

\section{References}

Artamonov, B. P., Badan, Yu. Yu., Bruevich, V. V., \& Gusev, A. S. 1999, Astron. Rep., 43, 377

Ashman, K. M., Bird, C. M., \& Zepf, S. E. 1994, AJ, 108, 2348

Buta, R. J. 2017, MNRAS, 470, 3819

Buta, R. J., Sheth, K., Athanassoula, E., et al. 2014, ApJS, 217, 32

Comerón, S., Salo, H., \& Laurikainen, E. 2014, A\&A, 562, A121

Díaz-Garcíaa, S., Salo, H., Knapen, J.H., \& Herrera-Endoqui, M. 2019, A\&A, 631, A94

Efremov, Yu. N. 2009, Astron. Lett., 35, 507

Efremov, Yu. N. 2010, MNRAS, 405, 1531

Efremov, Yu. N. 2011, Astron. Rep., 55, 108

Elmegreen, B. G. 1994a, ApJ, 425, L73

Elmegreen, B. G. 1994b, ApJ, 433, 39

Elmegreen, B. G., \& Elmegreen, D. M. 1983, MNRAS, 203, 31

Elmegreen, B. G., Elmegreen, D. M., Chromey, F. R., Hasselbacher, D. A., \& Bissell, B. A. 1996, AJ, 111, 2233

Elmegreen, B. G., Elmegreen, D. M., \& Efremov, Yu. N. 2018, ApJ, 863, 59

Epinat, B., Amram, P., \& Marcelin, M. 2008, MNRAS, 390, 466

Font, J., Beckman, J. E., Querejeta, M., et al. 2014, ApJS, 210, 2

Font, J., Beckman, J. E., James, P. A., \& Patsis, P. A. 2019, MNRAS, 482, 5362

Garrido, O., Marcelin, M., Amram, P., et al. 2005, MNRAS, 362, 127

Gusev, A. S., \& Efremov, Yu. N. 2013, MNRAS, 434, 313

Gusev, A. S., Guslyakova, S. A., Novikova, A. P., et al. 2015, Astron. Rep., 59, 899

Gusev, A. S., Shimanovskaya, E. V., Shatsky, N. I., et al. 2018, Open Astron., 27, 98

Hernandez, O., Carignan, C., Amram, P., Chemin, L., \& Daigle, O. 2005, MNRAS, 360, 1201

Horne, J. H., \& Baliunas, S. L. 1986, ApJ, 302, 757

James, P. A., Shane, N. S., Beckman, J. E., et al. 2004, A\&A, 414, 23

Morrissey, P., Schiminovich, D., Barlow, T. A., et al. 2005, ApJ, 619, L7

Press, W. H., \& Rybicki, G. B. 1989, ApJ, 338, 277

Scargle, J. D. 1982, ApJ, 263, 835

VanderPlas, J. T. 2018, ApJS, 236, 16

van Driel, W., \& Buta, R. J. 1991, A\&A, 245, 7

Véron-Cetty, M. P., \& Véron, P. 2010, A\&A, 518, A10

Whitmore, B. C., Chandar, R., Kim, H., et al. 2011, ApJ, 729, 78

Williams, D. R. A., McHardy, I. M., Baldi, R. D., et al. 2019, MNRAS, 486, 4962 